\title{
Diagnostic yield of CT pulmonary angiography in the diagnosis of pulmonary embolism: A single center experience
}

\begin{abstract}
The purpose of this study was to investigate the clinical application of computed tomography pulmonary angiography (CTPA) in patients with suspected pulmonary embolism (PE) based on a single-center experience. Medical records and radiological reports of 450 patients ( 272 men and 178 women; mean age 58.9 years; range $14-95$ years) with suspected PE who underwent CTPA and triple rule-out CT scans over a period of 6 months were retrospectively reviewed. Prevalence of $\mathrm{PE}$ was analysed to determine the diagnostic yield of CT in these two scanning protocols. Of 450 patient records, the positive rate of PE was $30.7 \%$. Triple rule-out CT protocol was performed in 75 out of 450 patients with the diagnostic yield of PE being only $8 \%$ ( 6 out of 75 patients had PE), which is significantly lower than the $35.2 \%$ as shown in the CTPA group (132 out of 375 had PE). Mean effective dose was $2.8 \pm 2.6 \mathrm{mSv}$ and $13.5 \pm 7.8 \mathrm{mSv}$ for CTPA and triple rule-out protocols, respectively. This study shows that CTPA has high diagnostic yield in the diagnosis of pulmonary embolism, hence justifying its appropriateness as a routine imaging modality. However, triple rule-out $\mathrm{CT}$ is not recommended due to the low diagnostic yield. Further reduction in radiation and contrast medium doses is necessary.
\end{abstract}

Keywords: Computed tomography pulmonary angiography - Diagnosis - Pulmonary embolism

\section{Introduction}

Computed tomography pulmonary angiography (CTPA) is currently the first line imaging technique as recommended by guidelines in the diagnosis of patients with suspected pulmonary embolism (PE) [1,2]. Estrada-Martin and Oldham developed surveys to determine different opinions among radiologists towards the use of CTPA for the diagnosis of $\mathrm{PE}$, and their results showed that most radiologists chose CTPA as the gold standard to diagnose PE [3]. The agreement with this statement is now based on the evidence available in many studies showing that high diagnostic accuracy has been achieved with CTPA in detecting thrombus in both main and side pulmonary artery branches due to rapid technological developments in CT scanning techniques
[4-11]. Another important advantage of CT angiography lies in its ability of examining other structures in the thoracic CT scans in addition to the detection of PE, such as assessment of aortic disease and coronary artery disease which is referred to as triple rule-out protocol due to similar symptoms of chest pain presented by these diseases to the emergency department $[12,13]$. Despite the benefit, a systematic review and meta-analysis has shown that there is insufficient evidence to recommend the use of triple rule-out in the diagnosis of PE due to low prevalence of PE $(<1 \%)[14]$.

Increased use of CTPA in the diagnosis of $\mathrm{PE}$ has raised concerns in the literature due to radiation exposure associated with CT and potential risk of contrast-induced nephropathy $[15,16]$. Further, no guidelines
Zhonghua Sun ${ }^{1 *}$ and Jing Lei ${ }^{2}$ 'Department of Medical Radiation Sciences, Curtin University, Perth, Western Australia, 6845, Australia ${ }^{2}$ Department of Medical Imaging, First Affiliated Hospital of Kunming Medical University, Yunnan, 650032, China

*Author for correspondence:

Tel: +61892667509

Fax: +61892662377

E-mail: z.sun@curtin.edu.au Submitted: August 18, 2017 Accepted: September 13, 2017 Published online: September 18, 2017 
are available about the minimum acceptable diagnostic yield of CTPA, thus leading to overuse of CTPA as a routine diagnostic tool $[17,18]$. Sharma and Lucas reported the direct correlation between CTPA and positive rates of $\mathrm{PE}$ detection with results justifying the increased use of CTPA [19]. However, Mountain et al in their multi-center study showed significant variations in the diagnostic yield of CTPA with some clinical sites producing lower diagnostic yield of less than $15.3 \%$ [20]. Due to these controversial reports and potential risks of radiation dose and use of contrast medium, it is necessary to further determine whether CTPA should be used as a routine diagnostic tool in the detection of PE.

The primary aim of this study was to analyse the diagnostic performance of CTPA in patients with suspected $\mathrm{PE}$, based on a single center experience. In addition to the routine use of CTPA, triple rule-out protocol was also analysed since patients presented with symptoms of chest pain may suffer from acute coronary syndrome, aortic dissection and PE. Thus, our secondary aim of the study was to determine the usefulness of triple rule-out CT protocol in the diagnostic assessment of PE.

\section{Materials and Methods}

\section{Patient data collection}

This retrospective study consisted of 450 patients (272 men and 178 women; mean age 58.9 years; range 14-95 years) with suspected symptoms of chest pain and difficulty in breathing who underwent CTPA or triple rule-out CT examinations within a 6 -month period in a tertiary hospital. Patients were referred by physicians for CT scans with the aim of detecting and diagnosing pulmonary embolism. Patients were excluded if CT scans could not complete due to allergic reactions to contrast medium and renal insufficiency, or suboptimal diagnostic image quality. Ethical approval was waived due to the retrospective nature of this study and the use of CT scans as part of routine clinical diagnosis.

\section{CT pulmonary angiography and triple rule-out scanning protocols}

All patients were scanned on a second generation of dual-source CT scanner (Siemens Definition Flash, Siemens Healthcare, Forchheim, Germany). The scanning protocol for CTPA was as follows: detector collimation $128 \times 0.6 \mathrm{~mm}$, gantry rotation $0.28 \mathrm{~s}$, tube voltage of $100 \mathrm{kVp}$ with automatic tube current modulation and pitch 1.45 . Slice thickness was 8 $\mathrm{mm}$ with reconstruction thickness and reconstruction interval of $1 \mathrm{~mm}$ and $0.7 \mathrm{~mm}$, respectively. The scanning protocol for triple rule-out CT was as follows: same detector collimation and gantry rotation as shown in the CTPA protocol, 80 to $120 \mathrm{kVp}$ with automatic tube current modulation and pitch 0.3 . Slice thickness was 8 $\mathrm{mm}$ with reconstruction thickness and reconstruction interval of $0.75 \mathrm{~mm}$ and $0.5 \mathrm{~mm}$ for coronary artery imaging, $1 \mathrm{~mm}$ and $0.7 \mathrm{~mm}$ for pulmonary artery and aorta imaging, respectively.

Non-ionic contrast medium Iopromide at $370 \mathrm{mg} /$ $\mathrm{ml}$ (Iopromide 370, Bayer Schering Pharma) was administered using a dual-head power injector. The scan was initiated using a bolus tracking technique with a CT attenuation of $150 \mathrm{HU}$ as the triggering threshold in the main pulmonary artery. Fifty to seventy $\mathrm{ml}$ of contrast medium was injected intravenously at a flow rate of $4-5.5 \mathrm{ml} / \mathrm{s}$, depending on the $\mathrm{kVp}$ used in the scanning protocols, followed by a saline chaser of 30 $\mathrm{ml}$.

\section{Data extraction and analysis}

Radiology reports and medical records were reviewed to identify the prevalence of $\mathrm{PE}$ (positive rates) among all of the CT scans, as well as the diagnostic yield in the two different groups of patients who underwent CTPA and triple rule-out CT scans separately. Further, radiation dose values in terms of volume CT dose index (CTDIvol), dose length product (DLP) which was available on the CT console were compared for each scan. Effective dose was calculated using a conversion factor of 0.014 [21].

\section{Statistical analysis}

All data were entered into SPSS 24.0 (SPSS Inc, Chicago., IL, USA) for statistical analysis. Continuous variables were expressed as mean \pm standard deviation, while categorical variables were presented as percentages or frequencies. Chi-square or Fisher's Exact test was used for analysis of categorical variables. A Student $T$ test was used to determine the differences between radiation dose between CTPA and triple rule-out protocols. A p value of less than 0.05 indicated statistically significant.

\section{Results}

Patient demographic characteristics are shown in Table 1. Of 450 patients, the presence of thrombus was found in at least one of the pulmonary arterial branches in 138 patients with positive rate of PE being $30.7 \%$. Of the positive cases, bilateral multiple pulmonary emboli were diagnosed in 59 cases (42.8\%). Chest pain is the most common symptom $(74 \%)$ in patients with positive $\mathrm{PE}$, with other symptoms presented in the 
Table 1: Patient characteristics.

\begin{tabular}{|lccc|}
\hline & \multicolumn{2}{c|}{ Table 1: Patient characteristics. } & \\
\hline Parameters & All patients $(\mathbf{n = 4 5 0})$ & CTPA group $(\mathbf{n = 3 7 5 )}$ & Triple rule-out group ( $\mathbf{n = 7 5 )}$ \\
\hline Age (yrs) & $58.9 \pm 15.9$ & $59.5 \pm 16.5$ & $56.2 \pm 12.6$ \\
\hline Gender (M/F) & $272 / 178$ & $226 / 149$ & $44 / 31$ \\
\hline CTDlvol (mGy) & $8.5 \pm 9.4$ & $5.4 \pm 4.0$ & $24 \pm 12.8$ \\
\hline DLP $(\mathrm{mGy} . \mathrm{cm})$ & $327.5 \pm 402.2$ & $198.7 \pm 185.9$ & $967.4 \pm 555.7$ \\
\hline Effective dose $(\mathrm{mSv})$ & $4.5 \pm 5.6$ & $2.8 \pm 2.6$ & $13.5 \pm 7.8$ \\
\hline Prevalence of PE & $138(30.7 \%)$ & $132(35.2 \%)$ & $6(8 \%)$ \\
\hline
\end{tabular}

Table 2: Clinical presentations in patients with diagnosed pulmonary embolism.

\begin{tabular}{|cc|}
\hline Symptoms in patients with positive PE (138) & Number of cases \\
\hline Chest pain & 102 \\
\hline PE follow-up & 5 \\
\hline Lower extremity abnormalities & 5 \\
$\quad$ Edema & 4 \\
Left lower limb venous thrombosis & 1 \\
Right lower limb venous thrombosis & 1 \\
Malignant tumours & 1 \\
Right lung cancer with brain metastasis & 1 \\
Ovarian cancer with abdominal pain and ascites & 1 \\
Right lower limb tumour & 1 \\
Postoperative procedures & 1 \\
Right hemicolectomy with lower lung atelectasis & 1 \\
Hysterectomy & 1 \\
Thyroidectomy & 1 \\
Post-operation of endometrial cancer & 1 \\
Coronary artery bypass grafting with lung infection & 1 \\
Other symptoms & 1 \\
Abnormal ECG & 2 \\
Headache & 1 \\
Epilepsy & \\
Coma & \\
Right lower limb pain & 1 \\
Left lower limb pain & 3 \\
Upper abdominal discomfort & 1
\end{tabular}

remaining 36 patients. Table 2 shows details of clinical presentations in patients with positive PE.

Seventy-five out of 450 patients were scanned with the triple rule-out CT protocol, with no significant difference in age $(\mathrm{p}=0.099)$ or gender distribution $(\mathrm{p}=0.933)$ between the triple rule-out and CTPA groups. However, the diagnostic yield of PE in the triple rule-out group was only $8 \%$ (6 out of 75 patients had $\mathrm{PE}$ ), which is significantly lower than the $35.2 \%$ as seen in the CTPA group (132 out of 375 had PE). Of 6 patients diagnosed with positive PE, 5 had the symptom of chest pain, and the remaining one developed lung infection following coronary artery bypass grafting.

Table 1 shows the mean value and standard deviation of CTDIvol, DLP and effective dose in all patients and the two groups scanned with CTPA and triple rule-out CT. The dose values from the triple rule-out CT group were significantly higher than those from the CTPA group $(\mathrm{p}<0.0001)$, indicating high radiation dose associated with the use of triple rule-out protocol.
Figure 1 is an example of a patient with bilateral pulmonary emboli detected on CTPA, while Figure 2 is an example of a patient with bilateral pulmonary emboli detected on triple rule-out CT scan. Figure 3 shows a normal CT scan without any sign of thrombus in the pulmonary arteries and normal aorta or coronary artery in a patient who underwent triple rule-out CT.

\section{Discussion}

This study analyses the diagnostic performance of CTPA in patients with suspected PE based on a single center experience and has two findings which are considered important from a clinical perspective: first, the diagnostic yield of CTPA is more than $30 \%$, which is above the acceptance level of $15 \%[17,18]$. This indicates the appropriate use of CTPA for routine diagnosis of PE. Second, triple rule-out CT protocol is associated with significantly higher radiation dose when compared to the CTPA protocol, but with low diagnostic yield of less than $10 \%$. Thus, it is not recommended to be used in the diagnosis of patients with PE. 

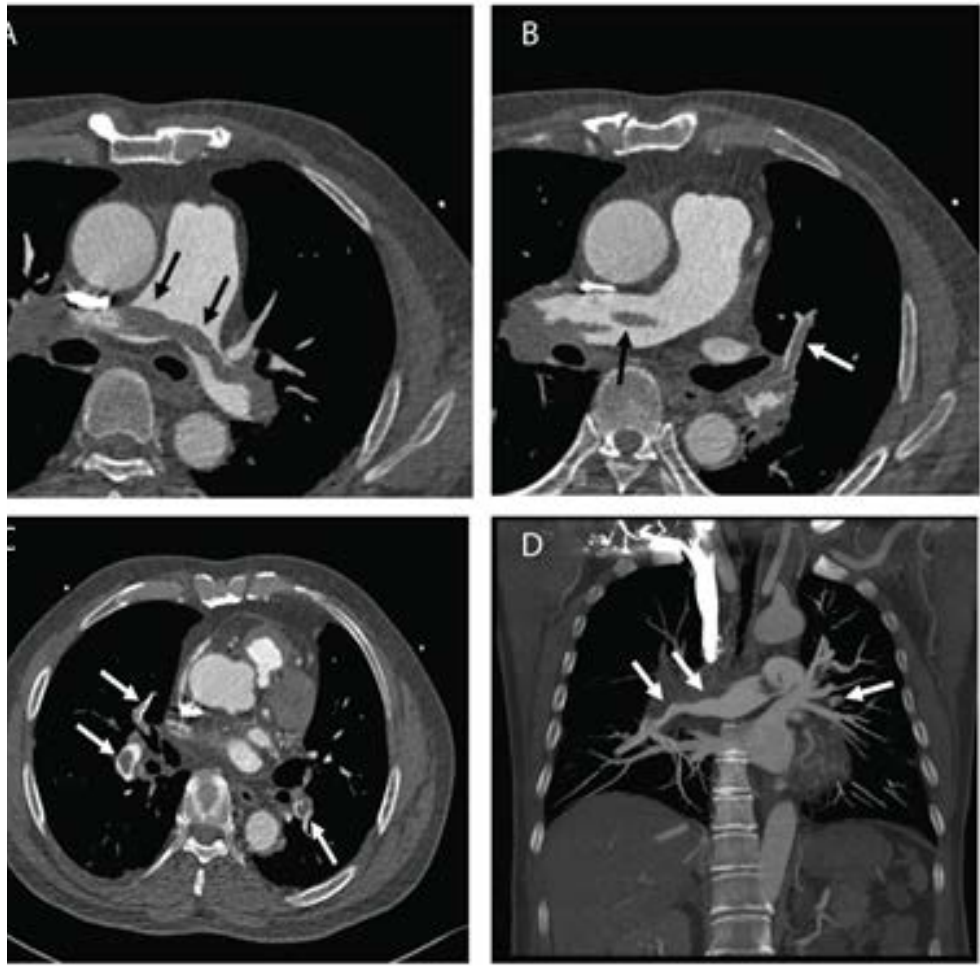

Figure 1: CT pulmonary angiography shows multiple pulmonary emboli in a 63-year-old male with chest pain for 7 days. $2 \mathrm{D}$ axial images show presence of thrombus in the main pulmonary arteries with extension to the segmental and subsegmental pulmonary arterial braches on both sides resulting in filling defects $(A-C)$. Coronal reformatted image demonstrates multiple emboli on pulmonary arteries (D). Arrows indicate the thrombi in pulmonary arteries.
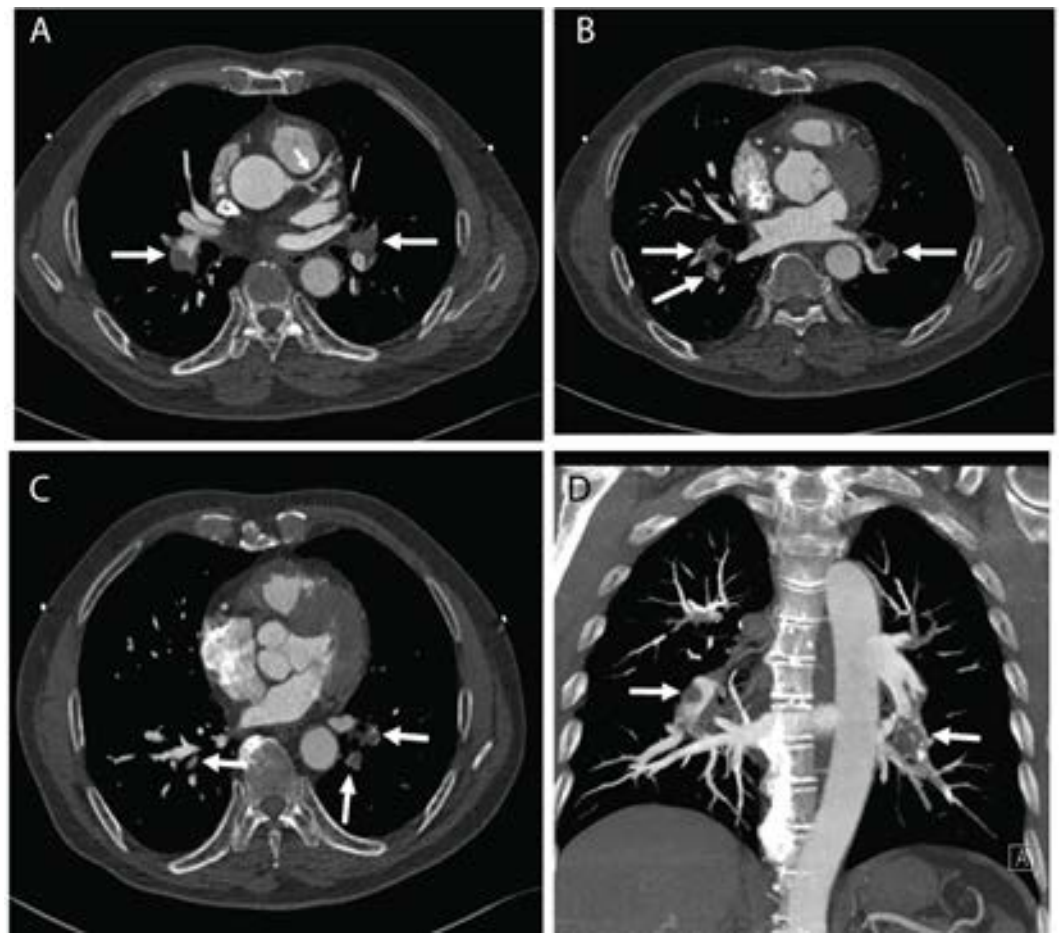

Figure 2: Triple rule-out CT scan shows multiple pulmonary emboli in a 65-yeare-old man with symptoms of coughing for 1 month. 2D axial images demonstrate thrombus present in the segmental and subsegmental pulmonary arteries on both sides (arrows in A-C). Coronal reformatted image reveals multiple emboli on pulmonary arterial branches (arrows in D). The left coronary artery is visualized as normal appearance (A). Long arrows indicate the thrombi in pulmonary arteries. Short arrow refers to the left coronary artery, which is normal. 

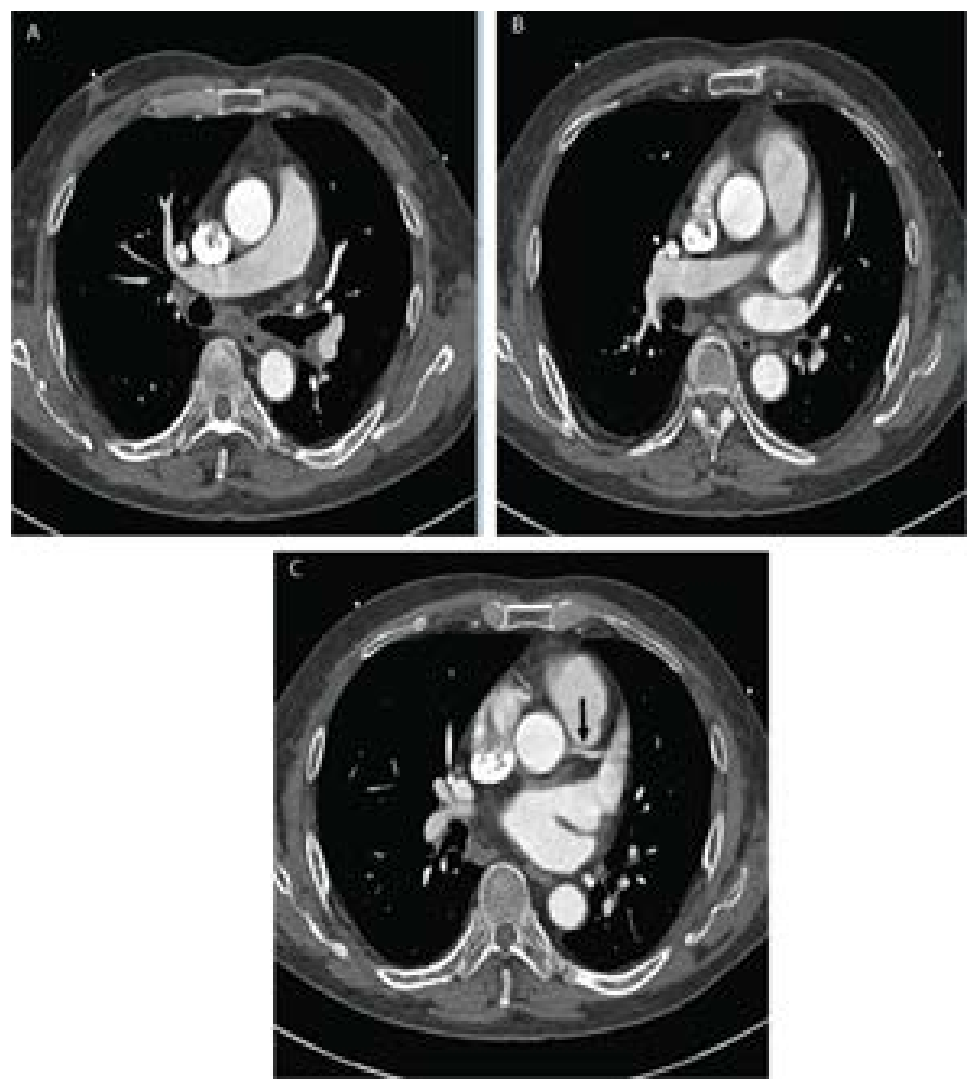

Figure 3: Triple rule-out CT shows normal findings in a 69-year-old man with chest discomfort. 2D axial images show normal pulmonary arteries without any sign of thrombus, or any abnormal changes to the thoracic aorta or coronary artery (A-C). Arrow points to the normal left coronary artery.

CTPA is currently the preferred imaging modality in the diagnosis of PE because of high diagnostic accuracy, less invasive nature and wide availability. Segmental and subsegmental PE can be reliably detected with latest $\mathrm{CT}$ scanners having superior spatial and temporal resolution [22,23]. Low-dose CTPA protocol has been increasingly used with significant reduction of radiation and contrast medium doses while still achieving acceptable diagnostic images [24-35]. This has led to the increasing use of CTPA in patients with suspected PE which raises the concern of inappropriately increased use of this examination in patients with low probability of PE. Hall et al reported that the prevalence of a new incidental finding during CTPA is high with the most common findings being pulmonary nodules (13\%) and adenopathy (9\%) [36]. These findings support an alternative diagnosis than to detect a PE because CTPA examinations obtained in the emergency department for diagnosis of PE were associated with more than twice as likely to find new incidental findings as to find PE (24\% incidental findings vs. 9\% PE). These new findings justify the clinical or radiological (such as CT) follow-up, thus, development of systematic approaches to determine clinical value and high diagnostic yield is necessary.

Chest pain is one of the most common reasons for patients to present to the emergency departments, as shown in this group of patients, however, the presenting signs and symptoms of $\mathrm{PE}$ are nonspecific and highly variable. Due to this reason, triple rule-out $\mathrm{CT}$ is increasingly used as a non-invasive technique in patients with chest pain to evaluate the coronary arteries, pulmonary arteries and thoracic aorta simultaneously for diagnosis of acute coronary syndrome, pulmonary embolism and aortic dissection [37-39]. However, there are some concerns about using the triple rule-out CT for diagnosis of PE due to high radiation dose but with low diagnostic yield. Rogg et al in their cross-sectional study showed that the diagnostic yield of triple ruleout CT for detection of PE and aortic dissection was $8.4 \%$ and $5.6 \%$, respectively [40]. Authors also found that of 626 visits to the emergency department, only $0.6 \%$ of patients were evaluated for these three diseases through triple rule-out CT. Ayaram et al. conducted a systematic review and meta-analysis of diagnostic accuracy of triple rule-out by analysing 11 studies [14]. 
Their analysis confirmed the high diagnostic accuracy of triple rule-out CT for detecting coronary artery disease with sensitivity and specificity more than $94 \%$. However, the prevalence of $\mathrm{PE}$ and aortic dissection was low $(<1 \%)$, thus the use of triple rule-out CT is not recommended in the diagnosis of these conditions. Our findings are consistent with these reports as the diagnostic yield of triple rule-out CT in PE is $8 \%$, but at a much higher radiation dose than the CTPA group. This further supports the statement of inappropriate use of triple rule-out protocol in patients with chest pain suspecting of $\mathrm{PE}$.

Our positive $\mathrm{PE}$ rate of $31 \%$ on all CT scans and $35 \%$ on CTPA scans are higher than the rates reported in other studies including the PIOPED II $[20,36,41,42]$. The multi-center PIOPED II trail reported the CTPA diagnostic yield of $23 \%$, and their CT scans were performed on older generations of 4-, 8- and 16-slice scanners [41]. Mountain et al. also conducted a multicenter study with inclusion of 14 clinical sites with 94\% of CTPA performed on 64-slice CT, indicating the use of recent CT scanners [20]. The overall diagnostic yield of CTPA at all sites was $14.5 \%$ (range: 9.3 to $25.3 \%$ ) with four sites having significantly lower yield which is less than the acceptable rate of $15.3 \%$. The high diagnostic yield in our study may be due to the use of second generation of dual-source CT scanner which shows improved detection rates, although this needs to be confirmed by further studies with inclusion of more patients and more clinical centers.

Some limitations exist in this study. First, this is a single-center experience, thus results may not be generalized to other clinical centers, in particular the high positive rates of PE detected in this cohort. Data in this study may suggest the improved diagnostic yield of CTPA in this region. Second, this study only focuses on the diagnostic yield of CTPA in the detection of $\mathrm{PE}$ without looking at other incidental findings such as new lung nodules or other thoracic changes that may have clinical importance or require further imaging follow-up. Further, in the subgroup analysis of patients undergoing triple rule-out CT, only detection of positive or negative $\mathrm{PE}$ was analysed while the diagnosis of other underlying diseases such as coronary artery disease or aortic dissection was not performed. Due to small sample size of patients undergoing triple rule-out CT (17\%) and very low prevalence of positive PE cases $(8 \%)$, conclusion about the lower diagnostic yield in the triple rule-out CT scan needs to be interpreted with caution. This limitation should be addressed in future studies. Third, although radiation dose in the CTPA group is similar to other studies, mean effective dose of $2.8 \mathrm{mSv}$ in this study is still much higher than the 0.9 $\mathrm{mSv}$ as reported in a recently published double low-dose CTPA protocol [28]. Thus, further dose reductions in both radiation exposure and contrast medium should be considered when CTPA is used as a routine imaging tool for diagnosis of $\mathrm{PE}$.

In conclusion, this single-center experience shows that the diagnostic yield of CTPA is more than $30 \%$, which is above the acceptable rate as suggested by the guidelines and other studies. This indicates that routine use of CTPA in the diagnosis of patients with pulmonary embolism is acceptable and appropriate in this group of patients. However, due to low diagnostic yield in the detection of pulmonary embolism, triple rule-out CT protocol is not recommended. Further studies are necessary to determine the diagnostic performance of CTPA in both thrombus detection and new incidental findings of lung disease. Further radiation dose and contrast medium dose reduction should also be considered with implementation of dose-reduction strategies.

\section{Executive summary}

This study was conducted to determine the diagnostic yield of CT pulmonary angiography (CTPA) in the diagnosis of pulmonary embolism based on a single center experience. Prevalence of pulmonary embolism was more than $30 \%$ in patients undergoing CTPA which is above the acceptable diagnostic yield, while prevalence of pulmonary embolism was only $8 \%$ in patients undergoing triple rule-out $\mathrm{CT}$.

This study further confirms the high diagnostic yield of CTPA in the diagnosis of pulmonary embolism, thus justifies its clinical value as a routine diagnostic tool. Triple rule-out CT is not recommended due to low diagnostic yield. 


\section{References}

1. Wells PS, Anderson DR, Rodger M, et al. Derivation of a simple clinical model to categorize patients probability of pulmonary embolism: increasing the models utility with the SimpliRED D-dimer. Thromb. Haemost. 83(3): 416-420 (2000).

2. NICE Clinical Guideline. Venous thromboembolic diseases: diagnosis, management and thrombophilia testing. NICE; 2012. Available from: https://www.nice.org.uk/guidance/ cg144/resources/venousthromboembolic- diseases-diagnosismanagement-and-thrombophilia-testing-35109570835141 (Accessed August 10, 2017).

3. Estrada-Y-Martin RM, Oldham SA. CTPA as the gold standard for the diagnosis of pulmonary embolism. Int. J. Comput. Assist. Radiol. Surg. 6(4): 557-563 (2011).

4. Mayo J, Thakur Y. Pulmonary CT angiography as first-line imaging for PE: image quality and radiation dose considerations. AJR. Am. J. Roentgenol. 200(3): 522-528 (2013).

5. Wittram C. How I do it: CT pulmonary angiography. AJR. Am. J. Roentgenol. 188(5): 1255-1261 (2007).

6. den Exter AL, van der Hulle T, Klok FA, Huisman MV. Advances in the diagnosis and management of acute pulmonary embolism. Thromb. Res. 133: S10-16 (2014).

7. Righini M, Le GG, Aujesky D, et al. Diagnosis of pulmonary embolism by multidetector $\mathrm{CT}$ alone or combined with venous ultrasonography of the leg: a randomised non-inferiority trial. Lancet. 371(9621): 1343-1352 (2008).

8. Ghanima W, Almaas V, Aballi S, et al. Management of suspected pulmonary embolism [PE] by D-dimer and multislice computed tomography in outpatients: an outcome study. J. Thromb. Haemost. 3(9): 1926-1932 (2005).

9. Mos IC, Klok FA, Kroft LJ, DE RA, Dekkers OM, Huisman MV. Safety of ruling out acute pulmonary embolism by normal computed tomography pulmonary angiography in patients with an indication for computed tomography: systematic review and meta-analysis. J. Thromb. Haemost. 7(9): 1491-1498 (2009).

10. Carrier M, Righini M, Wells PS, et al. Subsegmental pulmonary embolism diagnosed by computed tomography: incidence and clinical implications. A systematic review and meta-analysis of the management outcome studies. J. Thromb. Haemost. 8(8): 1716-1722 (2010).

11. Stein PD, Chenevert TL, Fowler SE, et al. PIOPED III (Prospective Investigation of Pulmonary Embolism Diagnosis III) Investigators. Gadolinium-enhanced magnetic resonance angiography for pulmonary embolism: a multicenter prospective study [PIOPED III]. Ann. Intern. Med. 152(7): 433-434 (2010).

12. Gruettner J, Fink C, Walter T, et al. Coronary computed tomography and triple rule out $\mathrm{CT}$ in patients with acute chest pain and an intermediate cardiac risk profile. Part I: impact on patient management. Eur. J. Radiol. 82(1): 100-105 (2013).

13. Krissak R, Henzler T, Prechel A, et al. Triple-rule out dualsource $\mathrm{CT}$ angiography of patients with acute chest pain: dose reduction potential of $100 \mathrm{kV}$ scanning. Eur. J. Radiol. 81(12): 3691-3696 (2012).

14. Ayaram D, Bellolio F, Murad H, et al. Triple rule-out computed tomographic angiography for chest pain: a diagnostic systematic review and meta-analysis. Acad Emerg. Med. 20(9): 861-871 (2013).
15. Mamlouk MD, vanSonnenberg E, Gosalia R, et al. Pulmonary embolism at CT angiography: implications for appropriateness, cost, and radiation exposure in 2003 patients. Radiology. 256(2): 625-32 (2010).

16. Mitchell AM, Jones AE, Tumlin JA, Kline JA. Prospective study of the incidence of contrast-induced nephropathy among patients evaluated for pulmonary embolism by contrastenhanced computed tomography. Acad. Emerg. Med. 19(6): 618-625 (2012).

17. Costantino MM, Randall G, Gosselin M, et al. CT angiography in the evaluation of acute pulmonary embolus. AJR. Am. J. Roentgenol. 191(2): 471-474 (2008).

18. Costa AF, Basseri H, Sheikh A, et al. The yield of CT pulmonary angiograms to exclude acute pulmonary embolism. Emerg. Radiol. 21(2): 133-141 (2014).

19. Sharma S, Lucas CD. Increasing use of CTPA for the investigation of suspected pulmonary embolism. Postgrad. Med. 129(2): 193-197 (2017).

20. Mountain D, Keijzers G, Chu K, et al. RESPECT-ED: rate of pulmonary embolism (PE) and sub-segmental $\mathrm{PE}$ with modern computed tomographic pulmonary angiograms in emergency departments; A multi-center observational study finds significant yield variation, uncorrelated with use or small PE rates. PloS. One. 11(12): e0166483 (2016).

21. McCollough $\mathrm{CH}$, Primak AN, Braun N, Kofler J, Yu L, Christner J. Strategies for reducing radiation dose in CT. Radiol. Clin. North. Am. 47(1): 27-40 (2009).

22. Chen EL, Ross JA, Grant C, et al. Improved I mage quality of low-dose CT pulmonary angiograms. J. Am. Coll. Radiol. 14(5): 648-653(2017).

23. Sun Z, Almoudi M, Cao Y. CT angiography in the diagnosis of cardiovascular disease: A transformation in cardiovascular CT practice. Quant. Imaging. Med. Surg. 4(5): 376-396 (2014).

24. Lu G, Luo S, Meinel FG, et al. High-pitch computed tomography pulmonary angiography with iterative reconstruction at $80 \mathrm{kVp}$ and $20 \mathrm{ml}$ contrast agent volume. Eur. Radiol. 24(12),32603268(2014).

25. Boos J, Kropil P, Lanzman RS, et al. CT pulmonary angiography: simultaneous low-pitch dual-source acquisition mode with $70 \mathrm{kVp}$ and $40 \mathrm{ml}$ of contrast medium and comparison with high-pitch spiral dual-source acquisition with automated tube potential selection. Br. J. Radiol. 89(1062): 20151059 (2016).

26. Kaur M, Vijayananthan A, Kumar G, Jayarani K, Ng KH, Sun Z. use of $100 \mathrm{kV}$ versus $120 \mathrm{kV}$ in computed tomography pulmonary angiography in the detection of pulmonary embolism: effect on radiation dose and image quality. Quant. Imaging. Med. Surg. 5(4): 524-533 (2015).

27. Faggioni L, Neri E, Sbragia P, et al. $80-\mathrm{kV}$ pulmonary CT angiography with $40 \mathrm{ml}$ of iodinated contrast material in lean patients: comparison of vascular enhancement with iodixanol $(320 \mathrm{mgl} / \mathrm{ml})$ and iomeprol $(400 \mathrm{mgI} / \mathrm{ml})$. AJR. Am. J. Roentgenol. 199(6): 1220-1225 (2012).

28. Laqmani A, Kurfurst M, Butscheidt S, et al. CT pulmonary angiography at reduced radiation exposure and contrast material volume using iterative model reconstruction and iDose 4 technique in comparison to FBP. PloS. One. 11(9): e0162429 (2016). 
29. Szucs-Farkas Z, Schibler F, Cullmann J, et al. Diagnostic accuracy of pulmonary CT angiography at low tube voltage: intraindividual comparison of a normal-dose protocol at 120 $\mathrm{kVp}$ and a low-dose protocol at $80 \mathrm{kVp}$ using reduced amount of contrast medium in a simulation study. AJR. Am. J. Roentgenol. 197(5): W852-859 (2011).

30. Szucs-Farkas Z, Schaller C, Bensler S, Patak MA, Vock P, Schindera ST. Detection of pulmonary emboli with CT angiography at reduced radiation exposure and contrast material volume: comparison of $80 \mathrm{kVp}$ and $120 \mathrm{kVp}$ protocols in a matched cohort. Invest. Radiol. 44(12): 793-799 (2009).

31. Laqmani A, Regier M, Veldhoen S, et al. Improved image quality and low radiation dose with hybrid iterative reconstruction with $80 \mathrm{kV}$ CT pulmonary angiography. Eur. J. Radiol. 83(10): 1962-1969 (2014).

32. Saade C, Mayat A, El-Merhi F. Exponentially decelerated contrast media injection rate combined with a novel patientspecific contrast formula reduces contrast volume administration and radiation dose during computed tomography pulmonary angiography. J. Comput. Assist. Tomogr. 40(3): 370-374 (2016).

33. De Zordo T, von Lutterotti K, Dejaco C, et al. Comparison of image quality and radiation dose of different pulmonary CTA protocols on a 128-slice CT: high-pitch dual source CT, dual energy CT and conventional spiral CT. Eur. Radiol. 22(2): 279-286 (2012).

34. Tacelli N, Remy-JardinM, Flohr T, et al. Dual-source chest CT angiography with high temporal resolution and high pitch modes: evaluation of image quality in 140 patients. Eur. Radiol. 20(5): 1188-1196 (2010).

35. Wichmann JL, Hu X, Kerl JM, et al. $70 \mathrm{kVp}$ computed tomography pulmonary angiography: potential for reduction of iodine load and radiation dose. J. Thorac. Imaging. 30(1): 69-76 (2015).

36. Hall WB, Truitt SG, Scheunemann LP, et al. The prevalence of clinically relevant incidental findings on chest computed tomographic angiograms ordered to diagnose pulmonary embolism. Arch. Intern. Med. 169(21): 1961-1965 (2009).

37. Burris AC, Boura JA, Raff GL, Chinnaiyan KM. Triple rule our versus coronary $\mathrm{CT}$ angiography in patients with acute chest pain: Results from the ACIC Consortium. JACC. Cardiovasc. Imaging. 8(7): 817-825 (2015).

38. Durmus T, Rogalla P, Lembcke A, et al. Low-dose triple-rule out using 320-row-detector volume MDCT-less contrast medium and lower radiation exposure. Eur. Radiol. 21: 1416-1423 (2011).

39. Kang EJ, Lee KN, Kim DW, et al. Triple rule-out chest pain evaluation using 320-row-detector volume CT: a comparison of the wide-volume and helical mode. Int. J. Cardiovasc. Imaging. 1: 7-13 (2012).

40. Rogg JG, De Neve JW, Huang C, et al. The triple work-up for emergency department patients with acute chest pain: how often does it occur? J. Emerg Med. 40(2): 128-134 (2011).

41. Stein PD, Fowler SE, Goodman LR, et al. PIOPED II Investigators. Multidetector computed tomography for acute pulmonary embolism. N. Engl. J. Med. 354(22): 2317-2327 (2006).

42. Brown MD, Vance SJ, Kline JA. An emergency department guideline for the diagnosis of pulmonary embolims: an outcome study. Acad. Emerg. Med. 12(1): 20-25 (2005). 